\title{
A Study on the Transmission Characteristics of the Human Body Towards Broadband Intra-body Communications
}

\author{
Jordi Agud Ruiz, Student Member, IEEE, Shigeru Shimamoto, Member, IEEE
}

\begin{abstract}
Intra-body communications is becoming a promising solution for Body Area Networks with a lot of interesting potential applications towards a ubiquitous communication world. Our research is based on the nearfield radio, wherein electromagnetic radio signals are transmitted through the human body from a transmitter to a receiver. In contrast with current studies, we aim to study the transmission characteristics of the human body as a conductor of radio signals with higher frequency carriers (up to $1400 \mathrm{MHz}$ ) in order to find an optimal frequency range for implementing, at last, broadband intra-body communications with high data rates. To achieve our first goal, we carry out experiments where we investigate the delay time profile and the attenuation of the received signal.
\end{abstract}

Index Terms - Intra-body communication, near-field radio, body area networks, propagation characteristics of human body.

\section{INTRODUCTION}

Body Area Networks (BAN) will allow new ways of wireless communications where the user will be able to interact with devices that move with the user or that are part of user's body [1]. Currently, there are several proposed technologies to achieve the goal of BAN. One of these solutions is intra-body communication (IBC), a short-range communication between devices in which the human body is used as the signal transmission medium.

The number of potential interesting applications by means of $\mathrm{IBC}$ is enormous. For example, users could transfer information between all sorts of electronic devices (mobile phones, PDAs, wearable devices such as wireless earpiece and eye-glass display...) by just touching or handling them. Moreover, IBC could be applied for new electronic money systems where users with an IBC enabled wristwatch with stored E-cash could purchase a product by just touching the product tag. Personal identification is another potential application where users could open the door of their houses by just touching the knob door. Even, at hospitals, doctors could know the medical record of the patient by just

The authors are with the Graduate School of Global Information and Telecommunication Studies, Waseda Uriversity, Shinjuku-ku, Tokyo, 1690051 Japan (e-mail: jordi.agud@toki.waseda.jp, shima@waseda.jp).

0-7803-8920-4/05/\$20.00 @ IEEE touching him, which it would be very useful in emergency situations.

Currently, there are two solutions for IBC: electric field type [2]-[5] and electromagnetic type [6]-[7]. By means of the former solution, data could be sent across devices placed on or near the body by using a near field electric field. In [2]-[4], electrical current used is lower than $\ln \mathrm{A}$, which it is safe for the hurnan body, frequency carrier is around $500 \mathrm{KHz}$ and the data rate achieved is up to $9.6 \mathrm{Kbps} .[2-4]$ employ an electrical sensor which it is characterized by a not very high input impedance, which makes accurate detection of signals through the body very difficult. In [5], the electric field sensor is implemented with electro-optic crystals and laser light. This kind of electro-optic sensor has high input impedance that it is useful for measurement of the unstable and small electric fields through the body. [5] achieves higher data rates and larger operating range then [2]-[4]. In the near-field radio IBC solution, the human body is treated as a waveguide with the high frequency electromagnetic signals generated at a terminal propagating through the body and being received by another terminal placed on the body. In [6] it is shown that data rates up to $9.6 \mathrm{Kbps}$ could be achieved using a frequency carrier of 10.7MHz.

Our research is based on the near-field radio. We aim to provide broadband IBC in order to satisfy future new applications (such as transferring multimedia files through the body) that will require high data rates and large bandwidths. For this reason, our first goal is to conduct several experiments to determine the transmission characteristics of the human body as a conductor of radio frequency signals - from $1 \mathrm{MHz}$ to $1400 \mathrm{MHz}$. Specifically, we aim to study the time delay profile and the attenuation of the received signal in this frequency range as a function of the distance between the transmitter (TX) and receiver (RX), the frequency carrier, the electrode size and the direction and configuration of the electrodes. Note that currently the maximum frequency carrier investigated for IBC is $40 \mathrm{MHz}$ [6] and $100 \mathrm{MHz}$ [7]. In the following section, we explain the general features of our IBC system model. In Section III, we explain the main features of the experiments that we have carried out in order to study the propagation characteristics of the human body. Afterwards, in Sections IV and $\mathrm{V}$, we show respectively the experimental results and the conclusions. 


\section{PROPOSED IBC SYSTEM MODEL}

Based on [1], the future mobile communications will evolve towards user-centred systems, which means that user will be in the centre interacting at different levels with different objects. We believe that mobile communications will be divided into two main groups: outside body (also called out-body) and on-body (also called IBC in this paper) communications. The former one can be subdivided into short-range (WLANs, pico-networks...) and long distance (cellular networks, satellite communications...) communications. As we explain into detail in [8], we believe that the future out-body communications will aim to provide the desired service based on users' interests and preferences at anytime and anyplace through the most suitable technology and operator's network available.

On-body communications can be divided into two subgroups: intra-body and inter-body communications. In this case, intra-body communications refer to the communication between devices attached to the same body, and inter-body communications refer to the case where communication is established between devices attached to different bodies that are in touch. Note that in this paper we use the general word IBC to refer to both intra-body and inter-body communications. On-body communications must aim to interconnect wearable devices (i.e. devices that move with the user or are part of user's body) and also to interact with user's surroundings.

\section{A. Elements and Features}

We differentiate three different kinds of elements/devices in our IBC system model: central unit (CU), mobile/wearable devices and, sensors and actuators. Fig. I shows an example of distribution of several gadgets in user's body. In this figure, the user is wearing the watchtype CU in his wrist, eye-glass display, earphones, mobile phone, a sensor for monitoring heart pulse (located close to the heart) and an environment sensing type gadget that allows users to

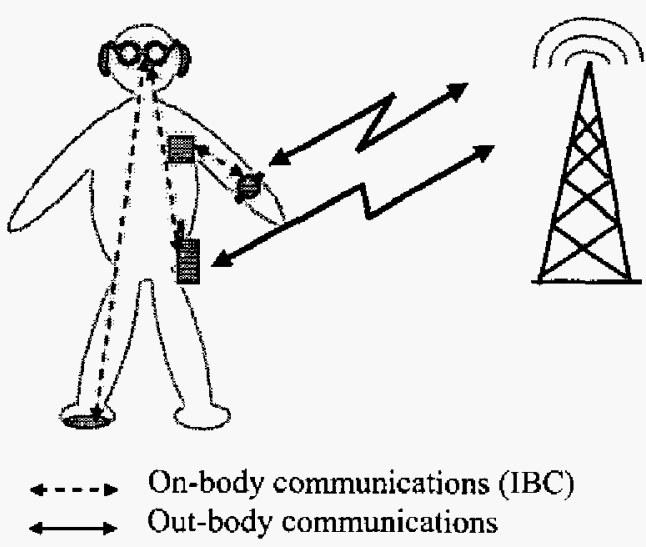

Fig. 1. Example of BAN devices distribution and type of communications receive information based on their location (e.g. in a department store, a customer can receive information of special prices or new products at each section while walking through his feet). All the devices in the Fig. 1 are IBC enabled. For example, the figure shows that the mobile phone transmits the data (e.g. a video mail) received from the base station to the eye-glass display in order to view the information in a high quality display.

$\mathrm{CU}$, that is the master device of user's BAN, could serve as an intelligent hub for user's BAN, managing all the active $\mathrm{BBC}$ enabled devices at anytime. CU could also store information about user's personal data (i.e. preferences, interests and identification passwords) and properties of the active IBC enabled devices. Moreover, CU must detect when user touches or grasps an IBC enabled device as this action could mean that user wants to establish an IBC. CU also must be able to transfer the data to the most suitable active device. That is, if the data is a multimedia file and the user's BAN is composed by the $C U$, a pager and an eyeglass display device, then the data should be sent to the eyeglass display device because of the better resolution of the display. Finally, CU should carry out not only on-body communications but also out-body communications, so that we ensure that at least one device of the user's BAN can communicate with the outside. We think that user's wrist could be a good place to mount on the CU.

The second group of the IBC system is mobile/wearable devices. This group is composed of devices such as mobile phone, PDA, notebook, eye-glass display, pager, MP3 player and wireless pen. Each gadget of this wide group is designed for a different purpose, so the features are also different (e.g. mobile phone can carry out voice communications but pager or PDA cannot). Some of these devices could carry out both on-body and out-body communications (e.g. mobile phone) and others only onbody communications (e.g. eye-glass display and MP3 player). We think that enabling all the devices to carry out on-body and out-body communications would represent an expensive and complex solution. Function-oriented devices that share $\mathrm{I} / \mathrm{O}$ module, out-body communication module, storage and computational resources would be the best solution to decrease the price and complexity of the hardware design. Therefore, as long as one device of the user's BAN has the capability for out-body communications, the rest of $\mathrm{IBC}$ devices can communicate with the outside, even if they are only on-body communicators, by using IBC with the on- out-body communicator. As an example, Fig. 1 shows that the $\mathrm{CU}$ and the mobile phone can perform both on-body and out-body communications and the rest of devices only on-body communications.

The third kind of elements that forms part of the IBC system is sensors and actuators. The function of these elements is respectively to collect data for the CU or another IBC enabled device, and to act based on some information 
from another device of user's BAN. Sensors and actuators are allowed to carry out only on-body communications.

\section{B. Communication Set $U p$}

As we have said previously, there are two types of IBC: $\mathrm{IBC}$ between a BAN device and the environment, and IBC between two or more BAN devices. In both cases the procedure to set up the communication is different. In the following subsection there is an example of communication between IBC enabled device and the environment. In this type of $I B C$, when the user touches an external IBC enabled object (EIO) of his surrounding, $\mathrm{CU}$ detects $\mathrm{EOI}$ and a communication link is established between them. Based on the type of data that EIO wants to transmit/receive and the properties of the active IBC enabled devices in user's BAN, $\mathrm{CU}$ decides which terminal is the most suitable and resends the data from EOI to the selected device. Because all the active BAN devices share the same transmission medium and in order to allow only the selected device to receive the data, each device has a unique identification code that is assigned by CU when the device accesses to the user's BAN. CU should send the identification code of the selected device together with the data so only the device that is identified by the code is allowed to receive the information properly.

The latter type of IBC is carried out mainly to transfer data from an IBC enabled device (source device, D1) to another one (candidate device, D2) which has better features for the type of data. When the user wants to run some data that it is already stored in D1 or D1 receives some data from the outside, before running this data in D1, the IBC system checks whether there is any available $\mathrm{D} 2$ which could be more suitable. In order to perform this process, firstly D1 establishes communication with $\mathrm{CU}$ to ask whether there is any D2 more suitable for the data willing to run. CU checks the list of active IBC enabled devices and if an available D2 is found, then CU sends to DI the identification code of the selected D2, so that D1 can transfer the data to D2. In case that CU doesn't find a more suitable IBC enabled device, the data is carried out in D1.

\section{Example Scenario}

In Fig. 2, we propose a new way of dynamic personalized advertising system by using IBC in trains. In Tokyo, the train coaches have above the handle a small plastic covering the strap of the handle (what we call advertising device, AD), where static ads are inserted (see Fig. 2). If this device has embedded an IBC enabled small system-on-a-chip where the company (e.g. travel agency) could store several type of data (such as text, audio and video) for several type of devices with commercial information addressed to different kind of potential customers (i.e. students, businessmen, retired people, ...), then, when the passenger touches the handle with his IBC enabled wristwatch, that is

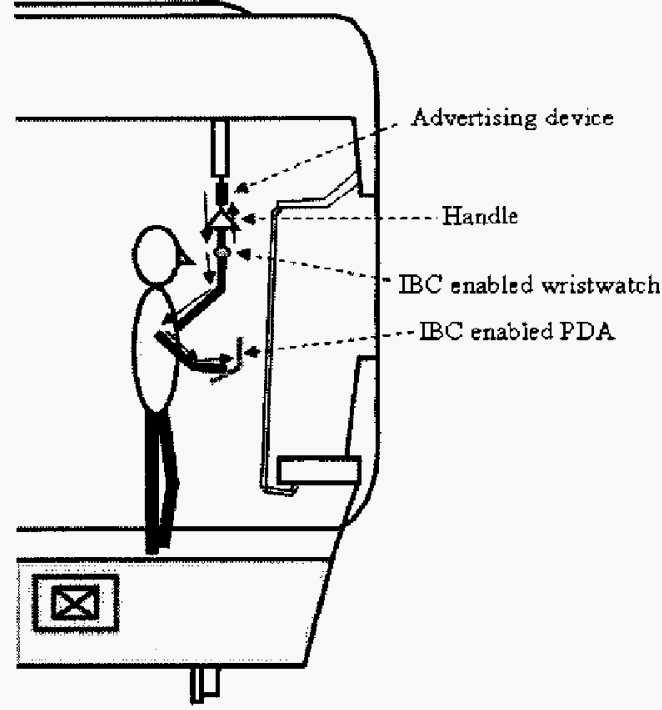

Fig. 2. Cross section of half train coach showing a potential new IBC application with personalized advertising

the CU of passenger's BAN, both $\mathrm{AD}$ and wristwatch could establish a communication. At the same time, the wristwatch could store some information about passenger's interests and preferences which could help the $A D$ to decide what kind of contents could be more suitable for the passenger, in case that he/she really wants to receive any information. The wristwatch, as the CU of user's BAN, manage all the BAN devices that user is carrying at each time and also their properties. So, depending on the type of BAN devices available, the wristwatch would ask the $A D$ for the most suitable type of data for the most suitable device and then it would resend it to this device (e.g. if a PDA, a mobile phone and a pager are available in the passenger's BAN, video data would be sent to the wristwatch, and then the wristwatch would resend it to a PDA because of the size of the display). Because all the active BAN devices share the same transmission medium, the wristwatch would resend the data with the identification code of the selected device, so that only the device which is identified by this code would be able to receive the information correctly.

\section{EXPERIMENT SETUP}

The experiments were performed in a classroom with desks and chairs. The Table I shows the main parameters of our experiments. IBC measurements were performed in the frequency range from 1 to $1400 \mathrm{MHz}$ with a frequency span TABLE I EXPEREMENT PARAMETERS

\begin{tabular}{l|l}
\hline Parameter & Value \\
\hline Frequency range [MHz] & $1 \sim 1400$ \\
\hline Frequency span [MHz] & 200 \\
\hline Frequency points & 1201 \\
\hline Input signa' power [dBm] & 7.0 \\
\hline Electrode diameter [cm] & $1,2,3$ \\
\hline Distance between TX and RX [cm] & 18,52 \\
\hline
\end{tabular}


of $200 \mathrm{MHz}$. A network analyzer is used to measure the time delay profile and the power of the received signals, our main aims at this stage. TX and RX have each one two round copper electrodes. One electrode is the signal electrode fed by an input signal generated by the network analyzer, and the other is the ground electrode (GND). Based on [9], the selected input signal power was $7 \mathrm{dBm}$, a safe power levelfor human body. In [9], some studies were carried out to investigate the possible health effects of exposure to electromagnetic fields (from $300 \mathrm{~Hz}$ to $300 \mathrm{GHz}$ ). These studies concluded that a basic limit exposure of $0.08 \mathrm{~W} / \mathrm{kg}$ was recommended for human body. Considering an average weight of $65 \mathrm{~kg}$, the maximum input signal power could be $37 \mathrm{dBm}$. Even so, we used a lower input power level of just $7 \mathrm{dBm}$. All the measurements were conducted for short $(18 \mathrm{~cm})$ and long $(52 \mathrm{~cm})$ distances between the electrodes of TX and RX, placed on the arm and the hand respectively. Fig. 3 shows the setting of the experiment carried out where TX is attached to the wrist and $\mathrm{RX}$ is touched by the fingers with a distance of $18 \mathrm{~cm}$ between TX and RX.

We took into consideration three different diameters of electrodes: $1 \mathrm{~cm}, 2 \mathrm{~cm}$ and $3 \mathrm{~cm}$. Based on [7], we also considered the direction of the electrodes of the TX: transverse and longitudinal direction. Moreover, we also investigated the performance in case of considering the TX with only signal electrode. For the configuration of the RX electrodes, we considered both a RX with signal and GND electrodes and another configuration without GND electrode (nGND).

\section{MEASUREMENT RESULTS}

One of the aims of the experiments is to study the attenuation of the received signals as a function of the electrode configuration and the frequency. Fig. 4 illustrates the average received power of the strongest echo as a

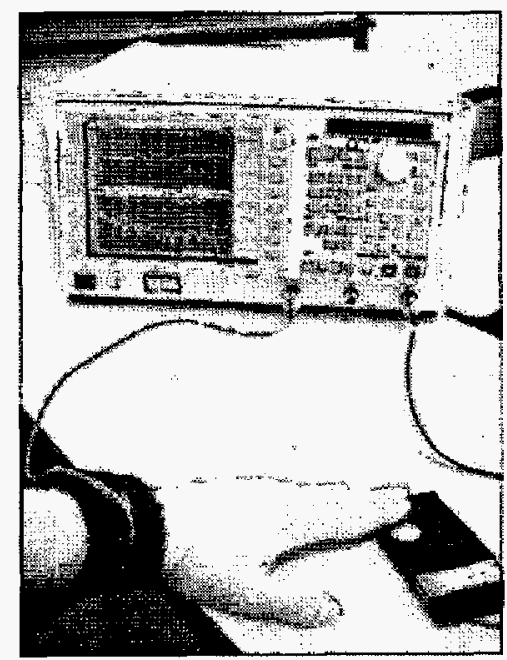

Fig. 3. Setting of the experiment s for IBC function of the six configurations of TX electrodes (transverse, longitudinal and TX without GND) and RX electrodes (with or without GND electrode), the electrode diameters and the distance between TX and RX. The average is calculated based on seven samples that were obtained from the frequency range of $1 \mathrm{MHz}$ to $1400 \mathrm{MHz}$ with a frequency span of $200 \mathrm{MHz}$. The results indicate that the lowest attenuation of the input signal is obtained in case of using a TX and a RX without GND for both short and long distances. In general, there is an average decay of $2.5 \sim 3.5 \mathrm{~dB}$ in all electrode configurations when increasing the distance from $18 \mathrm{~cm}$ to $52 \mathrm{~cm}$.

Figs. 5 and 6 show the received signal power as a function of the frequency in case of a distance between TX and $\mathrm{RX}$ of $18 \mathrm{~cm}$ and $52 \mathrm{~cm}$ respectively. In both figures there are six different curves that represent the received signal power of the six electrode configurations used in our experiments. The diameter of the electrodes in both figures is $2 \mathrm{~cm}$. The graphs show that the configuration of $\mathrm{TX}$ and $\mathrm{RX}$ without GND (nGND) is the one that performs the highest received signal power in all the frequency range for both distances. We also observe from the figures that, in all the cases, the attenuation increases when the frequency is increased.

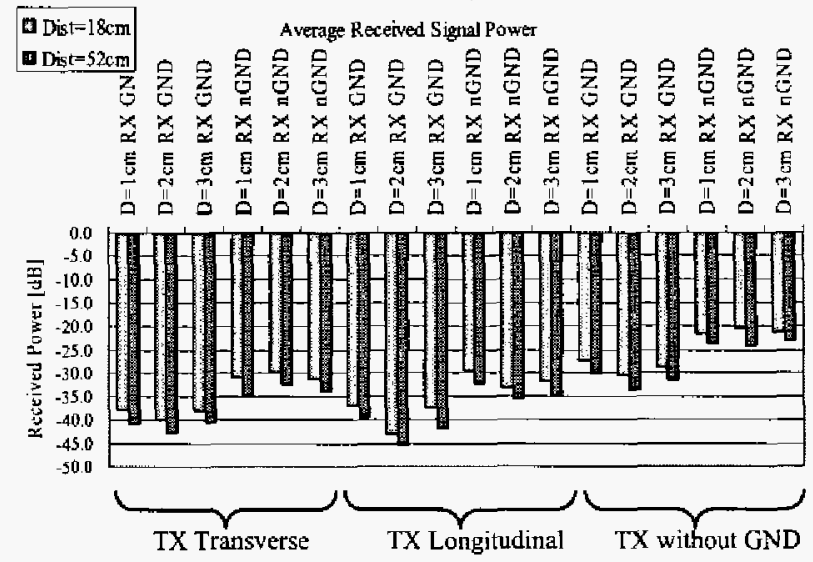

Fig. 4. Average received power of the strongest echo as a function of the electrode configuration, electrode size and distance between $\mathrm{TX}$ and $R X(18$ and $52 \mathrm{~cm})$

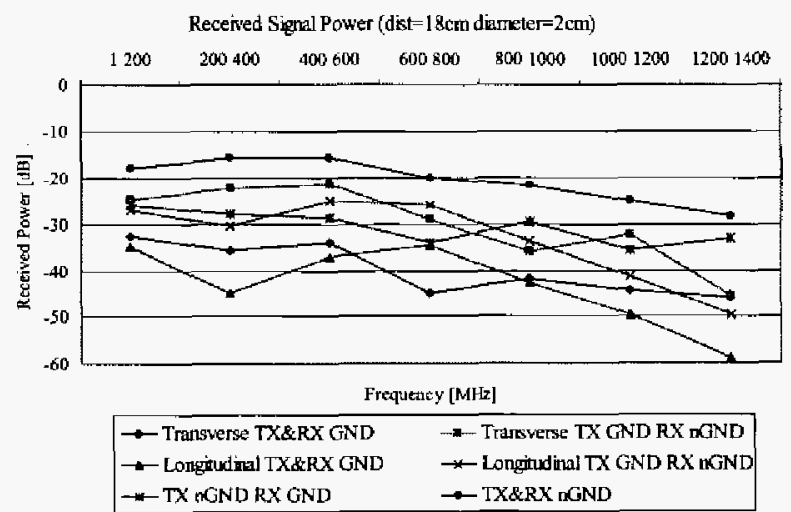

Fig. 5. Received signal power in case of electrode diameter of $2 \mathrm{~cm}$ and distance between $\mathrm{TX}$ and $\mathrm{RX}$ of $18 \mathrm{~cm}$ 


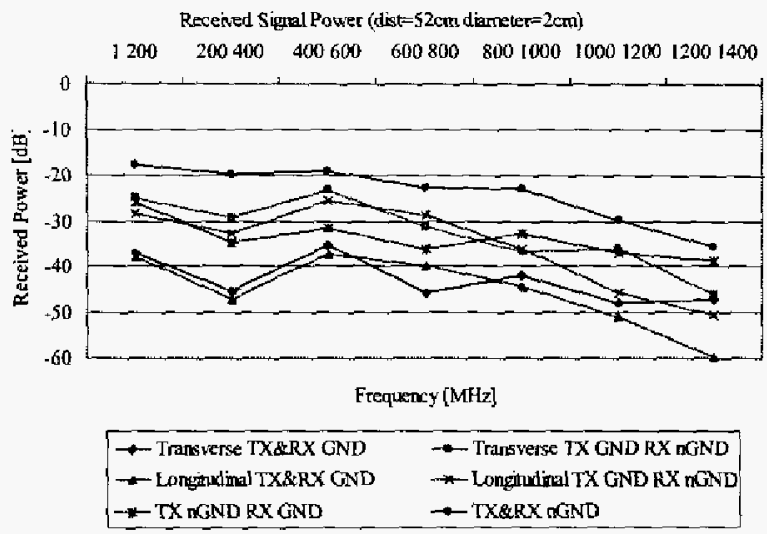

Fig. 6. Received signal power in case of electrode diameter of $2 \mathrm{~cm}$ and a distance between $\mathrm{TX}$ and $\mathrm{RX}$ of $52 \mathrm{~cm}$

The time delay profile is calculated by the inverse fourier transform from the measured frequency transfer functions (consisting of 1201 frequency points). Fig. 7 shows the average time delay of the strongest echo as a function of the direction of the TX electrodes (transverse, longitudinal and TX without GND), the configuration of the RX electrodes (with or without GND electrode), the diameter of the electrodes $(1 \mathrm{~cm}, 2 \mathrm{~cm}$ and $3 \mathrm{~cm}$ ) and the distance between TX and RX. The average is calculated based on seven samples that are obtained from the frequency range of $1 \sim 1400 \mathrm{MHz}$ with a frequency span of $200 \mathrm{MHz}$. The results show that the transverse configuration of $\mathrm{TX}$ electrodes provides the lowest average time delay. Moreover, the results also show that, in general, the electrodes with a diameter of $2 \mathrm{~cm}$ provide the best performance for all the different electrode configurations of $\mathrm{TX}$ and $\mathrm{RX}$.

Fig. 8 shows the time delay profile in case of considering TX and RX without GND electrode, diameter of $2 \mathrm{~cm}$, a frequency range between $1 \mathrm{MHz}$ and $800 \mathrm{MHz}$ and a distance of $18 \mathrm{~cm}$ between TX and RX. Y-axis represents the

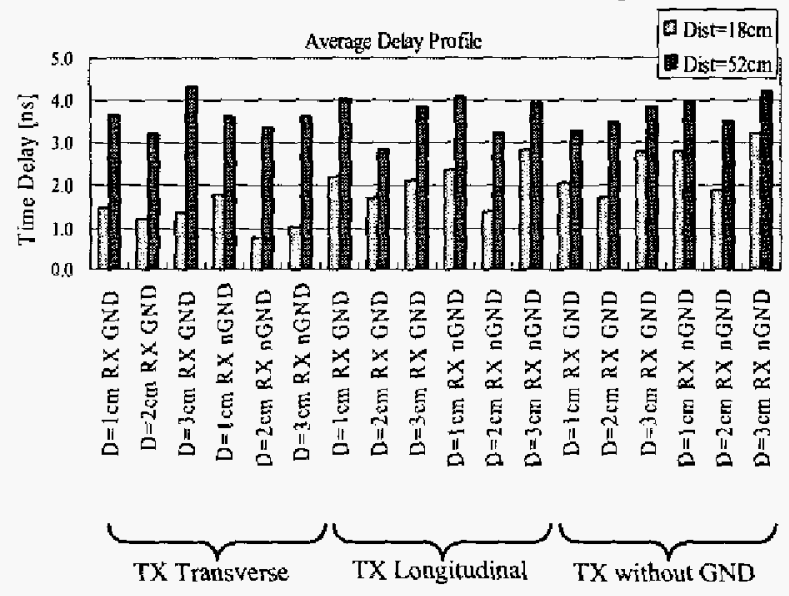

Fig. 7. Average time delay profile of the strongest echo as a function of the electrode configuration, electrode size and distance between $\mathrm{TX}$ and $\mathrm{RX}(18$ and $52 \mathrm{~cm})$

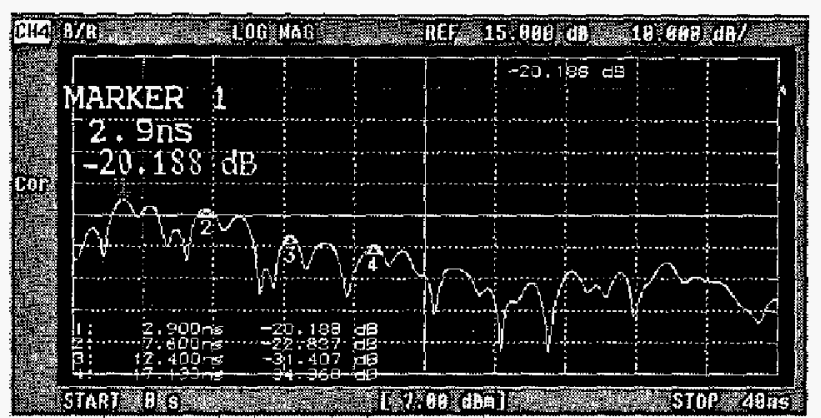

Fig. 8 . Time delay profile in case of ' $T X$ and $R X$ without GND electrode and a distance of $18 \mathrm{~cm}$ between $T X$ and $R X$

received signal power in $\mathrm{dB}$ and $\mathrm{x}$-axis represents the time delay in nanoseconds. The figure shows that the strongest echo takes $2.9 \mathrm{~ns}$ to reach the RX with a power of $-20.188 \mathrm{~dB}$. We also observe some periodicity between the first five echoes with a time difference of approximately 5 ns between echoes. Finally, the graph shows that there is a big attenuation of around $-14 \mathrm{~dB}$ between the 1st and 4 th echoes.

\section{CONClusion}

The experiments have shown that the optimal configuration is composed by $\mathrm{TX}$ and $\mathrm{RX}$ without GND electrodes and a diameter of $2 \mathrm{~cm}$ because of the lowest attenuation performed (note that the attenuation of the received signal is a critical parameter since the input signal is quite limited and weak in order to be safe for the human body). From a point of view of time delay, the configuration of TX and RX without GND does not provide the best performance. However, we still consider this electrode configuration as the most suitable one because the difference of results with regards to the transverse (the optimal solution in terms of time delay) and longitudinal configurations is not big. Finally, from the experiments, we believe that the frequency range between $1 \mathrm{MHz}$ to $600 \mathrm{MHz}$ could be the optimal as the power attenuation is lower than the case where higher frequencies are employed.

\section{References}

[1] Wireless World Research Forum, "Book of Visions 2001," Dec. 2001, www.wwrf.org

[2] T. G. Zimmerman, "Personal Area Networks: Near-filed Intra-Body Communication," IBM Systems Joumal, Vol .35, N. 3\&4, pp. 609617,1996

[3] E. Rehmi Post et al, "Intra-body Buses for Data and Power," Proc. of the Ist. Intemational Symposium on Wearable Computers, IEEE Comp. Soc. Press, 1997

[4] Matsushita Electric Works Ltd., 2004, www.mew.co.jp/epress/2004/0409-02.htm

[5] M. Shinagawa et al., "A Near-Field-Sensing Transceiver for Intrabody Communication Based on the Electrooptic Effect," IEEE Transactions on instrumentation and measurement. Vol. 53, NO. 6, december 2004

[6] K. Hachisuka Post et al., "Development and Performance Analysis of an Intra-Body Communication Device," Proc. of 12 th International Conference on Solid State Sensors, Actuators and Microsystems, Boston, pp. 1722-1725, June 2003 
[7] K.Fujii, K. Ito, "Evaluation of the Received Signal Level in Relation to the Size and Carrier Frequencies of the Wearable Device Using Human Body as a Transmission Channel," Proc. of 2004 Antennas and Propagation Society Symposium, pp. 105-108, June 2004

[8] J. Agud, S. Shimamoto. "Dynamic Mobile Operator Access Selection Schemes," Proc of 2004 Personal Mobile Communications Conference, Abano Terme (Italy), Seplember 2004

[9] World Health Organization, "Electromagnetic Fields $(300 \mathrm{~Hz}$ to 300GHz)," 1993, www inchem.org/documents/ehc/ehc/ehc 137.htm

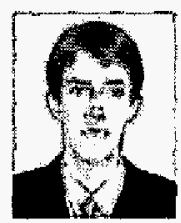

Jordi Agud Ruiz was born in Barcelona Spain, in 1974. He received the B.S. degree in Telecommunication Engineering from the Technical University of Catalonia, Vilanova i la Geltru, Spain, in 1998, and the M.S. degree in Global Information and Telecom Studies from Waseda University, Tokyo, Japan, in 2004. He is currently pursuing the Ph.D. degree in Global Information and Telecom Studies at Waseda University. His research interests are in the area of wireless communications, including future mobile cornmunication systems, short-range wireless communications, software defined radio and ultra wideband radio. He is a student member of IEEE and IEICE.

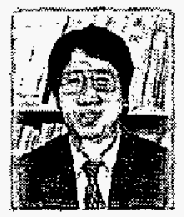

Shigeru Shimamoto received B.S. and M.S. degrees from the University of Electro-Communications, Tokyo, Japan in 198.5 and 1987 respectively, and Ph.D. degree from Tohoku University, Japan in 1992. From 1987 to 1991, he joined NEC Corporation, Japan. From 1991 to 1992, he was an assistant professor in the University of Electro-Communications. He joined Gunma University, Japan as an assistant professor in 1992, and promoted to associate professor in 1994. From 2000, he is professor in Waseda Unjversity, Japan. His research interests include wireJess communications, satellite telecommunication, stratospheric aircraft communication system, multiple access system, optical wireless communication, FWA and software architecture. He is a member of IEEE and IEICE. 